\title{
28 Research Square \\ Full-length MAVS Inhibits Hepatitis E Virus \\ Replication Dependent of JAK Signaling
}

\section{Changbo Qu}

Radboud Universiteit Nijmegen: Radboud Universiteit https://orcid.org/0000-0003-2909-156X

\section{Yang Li}

Erasmus Medical Centre: Erasmus MC

\section{Yunlong Li}

Erasmus Medical Centre: Erasmus MC

\section{Yihang Pan ( $\sim$ panyih@mail.sysu.edu.cn )}

SAHSYSU: The Seventh Affiliated Hospital Sun Yat-sen University

\section{Research Article}

Keywords: HEV, MAVS, IFNs, inhibition

Posted Date: August 3rd, 2021

DOl: https://doi.org/10.21203/rs.3.rs-765490/v1

License: (c) (1) This work is licensed under a Creative Commons Attribution 4.0 International License. Read Full License 


\section{Abstract}

Hepatitis E virus (HEV) infection is the leading cause of acute hepatitis worldwide. Mitochondrial antiviral signaling protein (MAVS)-mediated interferon (IFN) response plays a pivotal role in the hepatic antiviral immunity. However, little is known about the effects of overexpression of MAVS on HEV infection. Here, we studied the effects of FL-MAVS on HEV. We found that overexpression of FL-MAVS profoundly inhibited HEV replication. The overexpression of FL-MAVS is accompanied by the secretion of functional IFNs and transcriptional induction of interferon stimulated genes (ISGs). Furthermore, we showed that the anti-HEV effect of FL-MAVS is largely dependent of the JAK signaling activation.

\section{Main Text}

The mitochondrial antiviral signaling protein (MAVS) is an adaptor protein, localized at outer mitochondria membranes and at endoplasmic reticulum (ER)-mitochondria tethering interfaces, making the connection between mitochondria and innate immunity. Upon RNA virus infection, the viral genome is released into the cytoplasm to initiate viral protein biosynthesis. The double-stranded (ds) RNA acts as pathogen-associated molecular patterns (PAMPs) that is recognized by sensors (RIG-I or MDA5) in the host cell cytosol. The N-terminal caspase activation and recruitment domains (CARDs) of RIG-I and MDA5 bind to a CARD in the MAVS, resulting in MAVS aggregation and the subsequent activation of downstream signaling which triggers the production and secretion of interferons (IFNs) including IFN-a, IFN- $\beta$, and IFN- $\lambda$ [3]. IFNs bind to cell surface receptors and lead to the activation of Janus kinase and subsequent transcriptional induction of hundreds of interferon stimulated genes (ISGs), which are thought to be the ultimate antiviral effectors [14]. Acting as a vital adaptor for IFN signaling, MAVS shows specific interactions with different hepatitis viruses. Hepatits $C$ virus (HCV) NS3/4A protease blocks the interferon (IFN) response by cleaving the MAVS protein. In parallel, a product of Hepatitis A virus (HAV) polyprotein processing, 3ABC, targets MAVS for proteolysis [8]. Instead of directly provoking MAVS proteolysis or MAVS cleavage, Hepatitis E virus (HEV) has been reported to induce MAVS polymer, rendering the infected cells more resistant to exogenous IFNs $[8,17]$. The HEV-induced MAVS polymer represents a remarkable example of virus evolution albeit the role of ectopic overexpression of MAVS on HEV infection remains unclear. Full-length MAVS (FL-MAVS) is the main form of MAVS that increase the production of IFNs [9]. In this study, we investigated the effects of FL-MAVS on HEV. We found that FLMAVS overexpression significantly inhibited HEV replication. Mechanistically, we showed that the antiHEV effect of FL-MAVS is largely dependent of JAK activation.

To study the effect of FL-MAVS on HEV, genotype $3 \mathrm{HEV}$ infectious cell culture models was first employed. Successful overexpression of FL-MAVS was confirmed in Huh7.5-p6 model at mRNA and protein levels (Fig. 1A). Overexpression of FL-MAVS potently reduced the HEV RNA in Huh7.5-p6 model (Fig. 1B). The anti-HEV effect of FL-MAVS was further confirmed in PLC-p6 model (Fig. 1C). Moreover, a significant inhibition of HEV replication by FL-MAVS was also observed in HEV genotype 1 replicon model, suggesting a broader genotypic activity of FL-MAVS (Fig. 1D). Our previous studies have demonstrated that overexpression of RIG-I and MDA5 significantly inhibited HEV replication via 
stimulating the expression of a large panel of ISGs. Interestingly, the increased levels of ISGs induced by RIG-I and MDA5 are not associated with functional IFNs production $[6,14,15]$. In the present study, we found that overexpression of FL-MAVS also promotes an antiviral state by robustly increasing the expression of ISGs in Huh7.5-p6 models and PLC-p6 models (Fig. 2A and Supplementary Fig. 1A). However, we found that overexpression of FL-MAVS increased IFNs RNA in Huh7.5-p6 model (IFN $\beta$ and IFN $\lambda 1$ ) and PLC-p6 model (IFNa, IFN $\lambda 1$ and IFN $\lambda 2$ ) determined by qRT-PCR assay (Fig. 2B and Supplementary Fig. 1B). The above results prompt us to ask whether functional IFNs are secreted upon FL-MAVS overexpression. To answer this question, conditioned medium (supernatant) from the FL-MAVS transduced HEV cell culture models (Huh7.5-p6 and PLC-p6) were collected and two IFN sensitive assays (IFN functional assay and an HCV replicon-based bioassay) were performed [6, 14]. We found that FLMAVS overexpression resulted in functional IFNs secretion, as exemplified by the observation that the conditioned medium obtained from these models significantly increased ISRE activation (Fig. $2 \mathrm{C}$ and Supplementary Fig. 1C) and decreased HCV-related luciferase activity (Fig. 2D and Supplementary Fig. 1D). To further define the role of autocrine IFNs in HEV replication upon FL-MAVS overexpression, two IFNs binding inhibitors were used. B18R (which blocks IFNa/ $\beta$ receptor binding) and 136R (which preferentially blocks IFN $\lambda$ receptor binding) are poxvirus-derived soluble IFN antagonists that has been used to antagonize IFNs binding in Huh7 cell cultures [4]. We found that treatment with B18R or 136R slightly reversed the anti-HEV effect of FL-MAVS determined by qRT-PCR assay, indicating that the secreted IFNs might partly contribute to the anti-HEV activity of FL-MAVS (Fig. 2E and 2F). The secreted IFNs activates the JAK signaling pathway, which could be pharmacologically blocked by JAK inhibitor [6, 13]. We then sort to investigate whether JAK signaling pathway is involved in the anti-HEV activity of FLMAVS. We found that adding $10 \mu \mathrm{M}$ JAK inhibitor robustly reversed the anti-HEV effects FL-MAVS in HEV infectious and replicon models (Fig. $2 \mathrm{G}$ and $2 \mathrm{H}$ ), indicating that JAK signaling pathway is largely required for the FL-MAVS-mediated inhibition of HEV. Taken together, we discovered that overexpression of FLMAVS inhibited HEV replication. Mechanistically, the anti-HEV effect of FL-MAVS is mainly through JAK signaling.

Mitochondria are cellular organelles that participate in various metabolic activities as well as the regulation of innate immunity. MAVS serves as a vital adapter linking mitochondria to the innate immunity [12]. MAVS-mediated IFN response has been found to be inhibited by a variety of virus infections. For example, Zika virus nonstructural proteins, NS3 and NS2B3, were shown to negatively regulate IFN response by targeting MAVS [5]. PB1-F2 protein of H7N9 virus could inhibit innate immunity via preventing MAVS aggregation [1]. Besides that, a nonstructural protein of andes orthohantavirus (ANDV) was found to antagonize IFN response through reducing MAVS ubiquitination, a posttranslational mechanism of protein modification that mediating MAVS aggregation [10]. Most recently, SARS-CoV-2 membrane glycoprotein has been identified to negatively regulate innate immunity by impairing MAVS aggregation in HEK293 cells [2]. With respect to HEV, a previous study showed that HepG2 cells harboring HEV replicon yielded MAVS polymer compared to the control cells, suggesting a potential role of MAVS aggregation against HEV replication [17]. Classically, the aggregation of MAVS during virus infection results in section of IFNs, followed by activation of JAK signaling pathway which leads to increase of 
ISGs. The increased ISGs are thought to further enhance IFN production, which in turn leads to stronger activation of ISGs and restricts viral infection through positive feedback loops [15]. However, our previous studies showed that RIG-I and MDA5 inhibited HEV dispensable of IFNs secretion [6, 14]. This could be explained by the evidences showing that RIG-I directly restricts viral replication by inhibiting the binding of viral polymerase to viral RNA [11] and that RIG-I and MDA5 can displace viral proteins bound to dsRNA [16]. Extended from our previous findings, we here demonstrated that overexpression of FL-MAVS also potently inhibited HEV replication via stimulating ISGs production. The anti-HEV activity of FL-MAVS is largely dependent of JAK signaling. However, we found that overexpression of FL-MAVS dramatically increased IFN $\lambda$ RNA in both Huh7.5 and PLC-based HEV cell culture models. This is consistent with the previous studies revealing that HEV infection induced IFN $\lambda$ production as a host innate immune response $[7,17]$. In addition, we demonstrated that FL-MAVS overexpression result in IFNs secretion although it seems that the secreted IFNs only partly mediate the anti-HEV effects. In summary, our present study further bolsters the vital role of RIG-I/MDA5-MAVS signaling pathway against HEV replication. However, more information is required on the distinct mechanistic mode-of-action aspects of FL-MAVS in HEV replication. Moreover, the noncanonical antiviral mechanisms of this signaling pathway are largely elusive, thus deserving further clarification, although the IFNs-dependent mechanisms also need to be further investigated.

\section{Declarations}

Acknowledgements: The authors thank Dr. Pinghui Feng (Keck School of Medicine, University of Southern California, Los Angeles, California, United States of America) for kindly providing the FL-MAVS and empty vectors.

Funding: This research was supported by the research start-up Fund of the Seventh Affiliated Hospital Sun Yat-sen University (SAHSYSU) to C.Qu (No. ZSQYRSFPD0028).

Conflict of interests: The authors declare that they have no competing interests.

\section{References}

1. Cheung PH, Lee TT, Kew C, Chen H, Yuen KY, Chan CP, Jin DY (2020) Virus subtype-specific suppression of MAVS aggregation and activation by PB1-F2 protein of influenza A (H7N9) virus. PLoS Pathog 16:e1008611

2. Fu YZ, Wang SY, Zheng ZQ, Yi H, Li WW, Xu ZS, Wang YY (2021) SARS-CoV-2 membrane glycoprotein $M$ antagonizes the MAVS-mediated innate antiviral response. Cellular \& molecular immunology 18:613-620

3. Hou J, Han L, Zhao Z, Liu H, Zhang L, Ma C, Yi F, Liu B, Zheng Y, Gao C (2021) USP18 positively regulates innate antiviral immunity by promoting K63-linked polyubiquitination of MAVS. Nat Commun 12:2970 
4. Laidlaw SM, Marukian S, Gilmore RH, Cashman SB, Nechyporuk-Zloy V, Rice CM, Dustin LB (2017) Tumor necrosis factor inhibits spread of hepatitis $C$ virus among liver cells, independent from interferons. Gastroenterology 153:566-578.e565

5. Li W, Li N, Dai S, Hou G, Guo K, Chen X, Yi C, Liu W, Deng F, Wu Y, Cao X (2019) Zika virus circumvents host innate immunity by targeting the adaptor proteins MAVS and MITA. Faseb j 33:9929-9944

6. Li Y, Yu P, Qu C, Li P, Li Y, Ma Z, Wang W, de Man RA, Peppelenbosch MP, Pan Q (2020) MDA5 against enteric viruses through induction of interferon-like response partially via the JAK-STAT cascade. Antiviral Res 176:104743

7. Murata K, Kang JH, Nagashima S, Matsui T, Karino Y, Yamamoto Y, Atarashi T, Oohara M, Uebayashi M, Sakata H, Matsubayashi K, Takahashi K, Arai M, Mishiro S, Sugiyama M, Mizokami M, Okamoto H (2020) IFN- $\lambda 3$ as a host immune response in acute hepatitis $E$ virus infection. Cytokine 125:154816

8. Qu C, Zhang S, Li Y, Wang Y, Peppelenbosch MP, Pan Q (2019) Mitochondria in the biology, pathogenesis, and treatment of hepatitis virus infections. Rev Med Virol 29:e2075

9. Rayamajhi M, Miao EA, Moorman NJ (2014) miniMAVS, You complete me! Cell 156:629-630

10. Ren Z, Ding T, Zuo Z, Xu Z, Deng J, Wei Z (2020) Regulation of MAVS expression and signaling function in the antiviral innate immune response. Front Immunol 11:1030

11. Sato S, Li K, Kameyama T, Hayashi T, Ishida Y, Murakami S, Watanabe T, lijima S, Sakurai Y, Watashi K, Tsutsumi S, Sato Y, Akita H, Wakita T, Rice CM, Harashima H, Kohara M, Tanaka Y, Takaoka A (2015) The RNA sensor RIG-I dually functions as an innate sensor and direct antiviral factor for hepatitis B virus. Immunity 42:123-132

12. Tiku V, Tan MW, Dikic I (2020) Mitochondrial functions in infection and immunity. Trends in cell biology 30:263-275

13. Wang W, Wang Y, Qu C, Wang S, Zhou J, Cao W, Xu L, Ma B, Hakim MS, Yin Y, Li T, Peppelenbosch MP, Zhao J, Pan Q (2018) The RNA genome of hepatitis E virus robustly triggers an antiviral interferon response. Hepatology 67:2096-2112

14. Xu L, Wang W, Li Y, Zhou X, Yin Y, Wang Y, de Man RA, van der Laan LJW, Huang F, Kamar N, Peppelenbosch MP, Pan Q (2017) RIG-I is a key antiviral interferon-stimulated gene against hepatitis E virus regardless of interferon production. Hepatology 65:1823-1839

15. Xu L, Wang W, Peppelenbosch MP, Pan Q (2017) Noncanonical antiviral mechanisms of ISGs: Dispensability of inducible interferons. Trends Immunol 38:1-2

16. Yao H, Dittmann M, Peisley A, Hoffmann HH, Gilmore RH, Schmidt T, Schmidt-Burgk J, Hornung V, Rice CM, Hur S (2015) ATP-dependent effector-like functions of RIG-I-like receptors. Molecular cell 58:541-548

17. Yin X, Li X, Ambardekar C, Hu Z, Lhomme S, Feng Z (2017) Hepatitis E virus persists in the presence of a type III interferon response. PLoS Pathog 13:e1006417

\section{Figures}


A

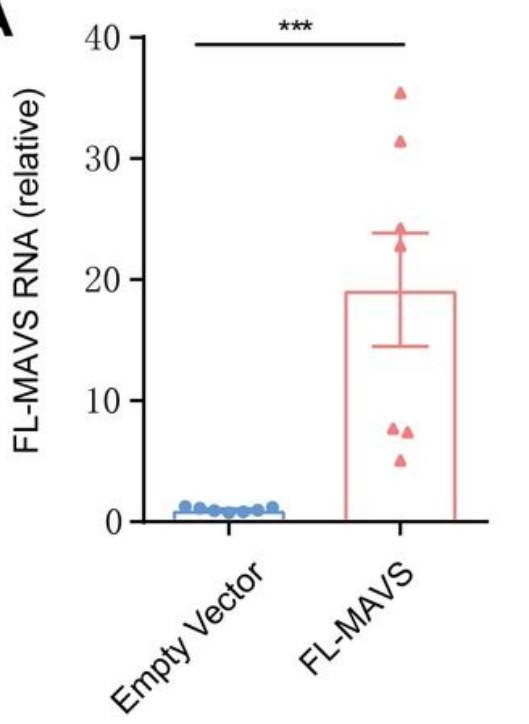

C

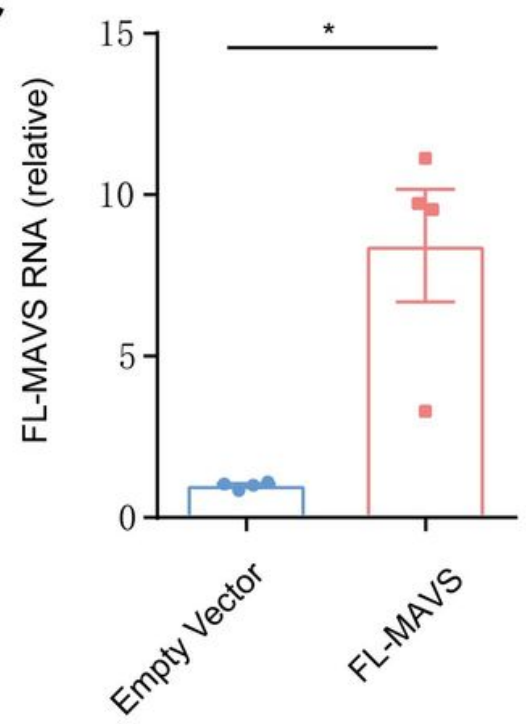

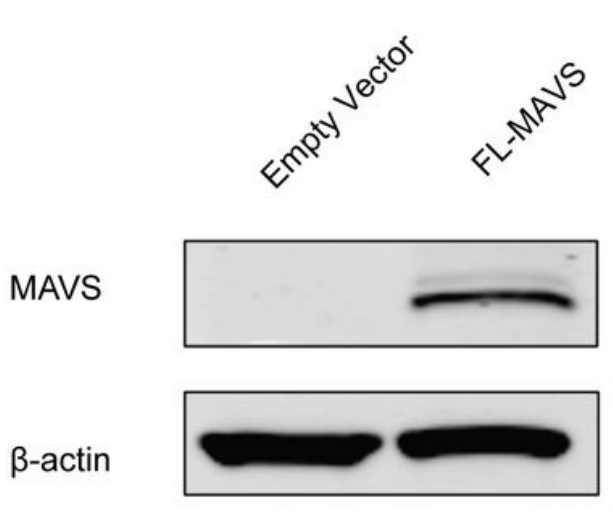
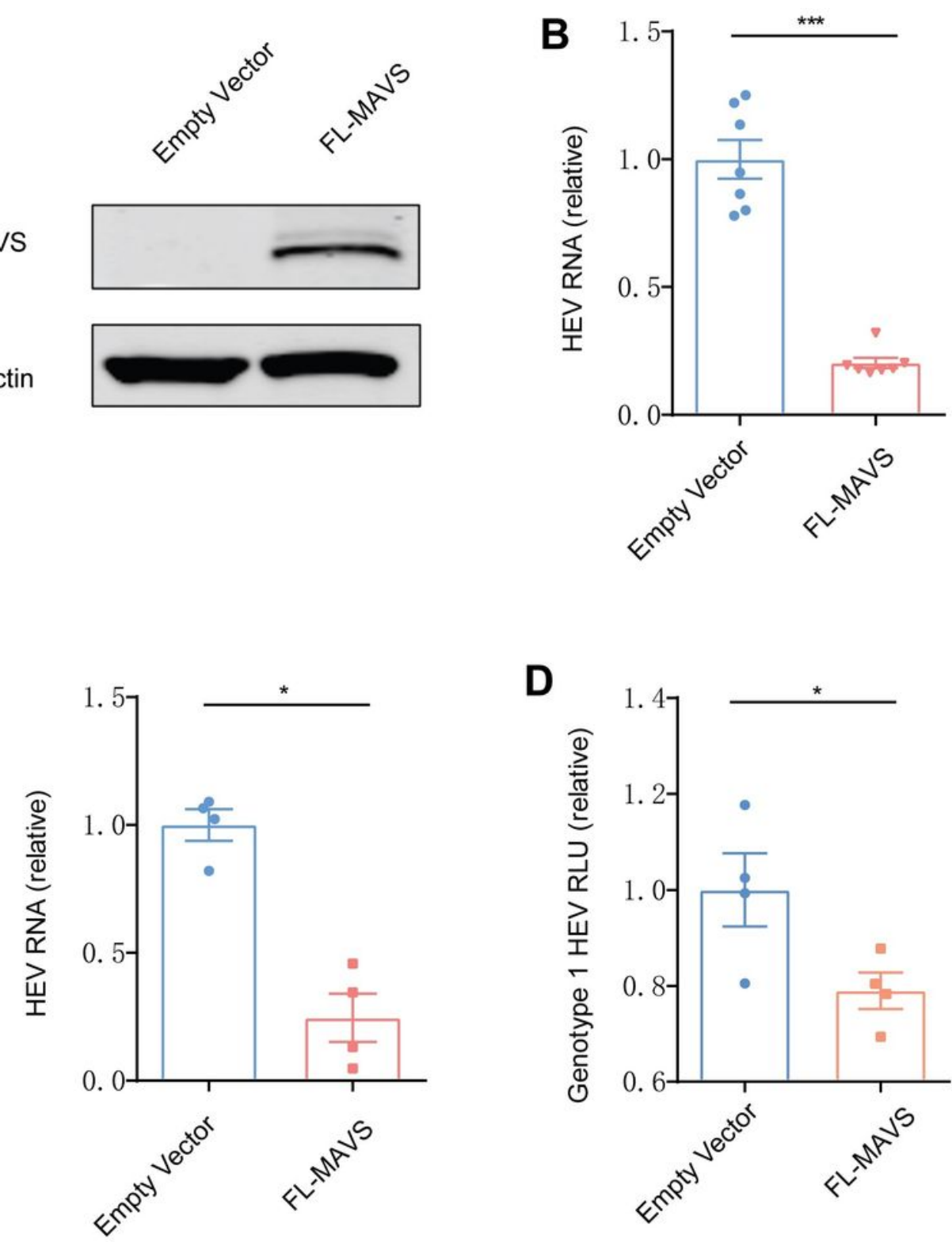

D

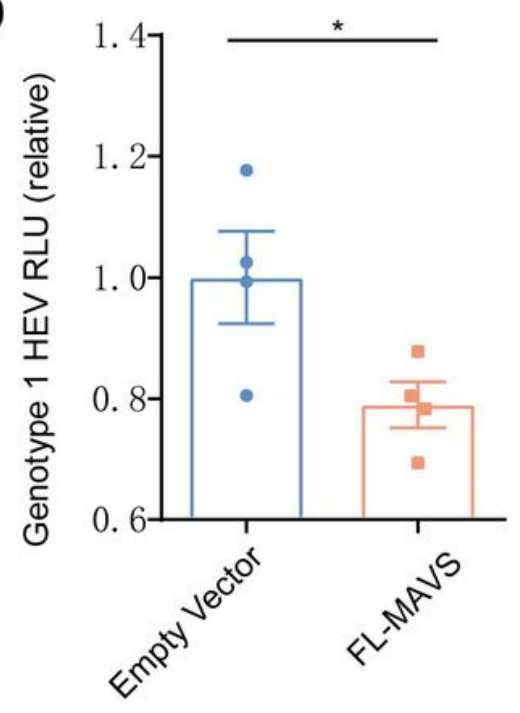

\section{Figure 1}

FL-MAVS inhibits HEV replication. Quantitative RT-PCR analysis of FL-MAVS RNA ( $n=7)$ and immunoblot analysis of FL-MAVS protein level using anti-FLAG antibody in Huh7.5-p6 cell model transduced with FLMAVS or empty vector (A). Quantitative RT-PCR analysis of HEV RNA $(n=7)$ in Huh7.5-p6 cell model transduced with FL-MAVS or empty vector for $48 \mathrm{~h}(\mathrm{~B})$. Quantitative RT-PCR analysis of FL-MAVS RNA $(n=4)$ and HEV RNA $(n=4)$ in PLC-p6 cell model transduced with FL-MAVS or empty vector (C) Analysis of HEV-related luciferase in genotype $1 \mathrm{HEV}$ cell model transduced with FL-MAVS or empty vector $(n=4)$ for $48 \mathrm{~h}$ (D). 
A

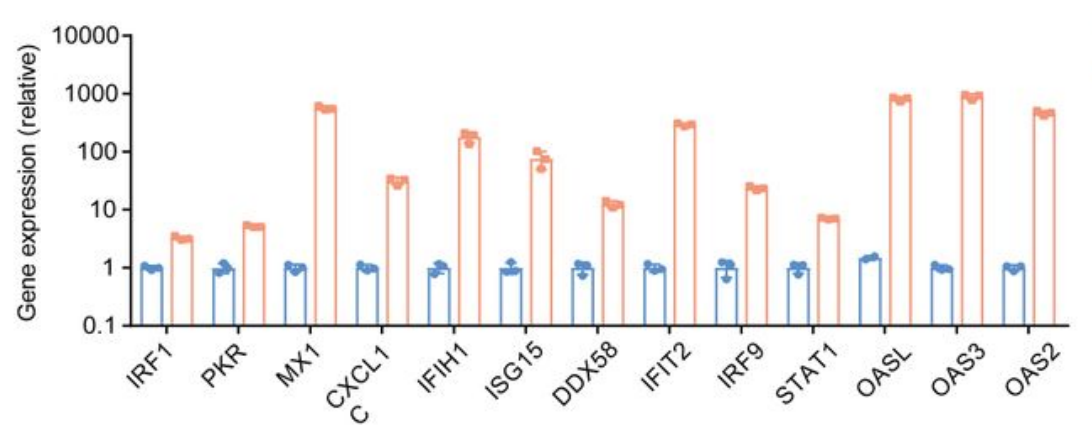

- Empty Vector

- FL-MAVS

B

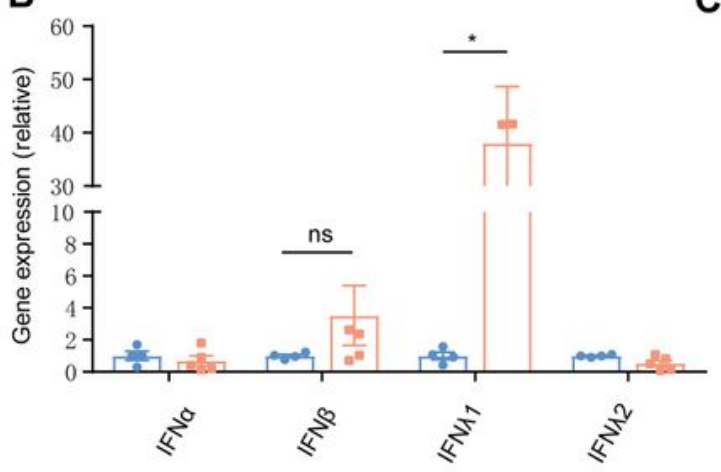

C

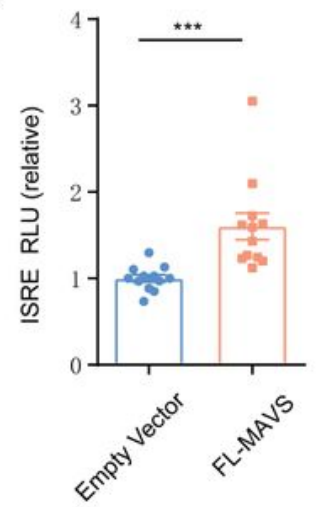

D

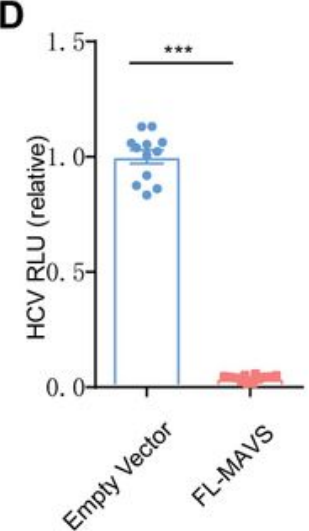

E

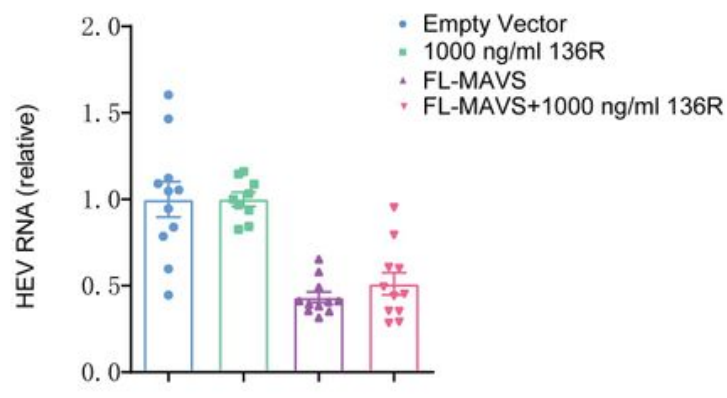

F

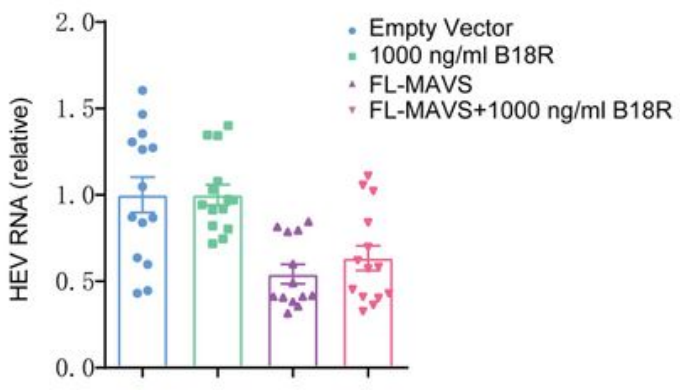

G

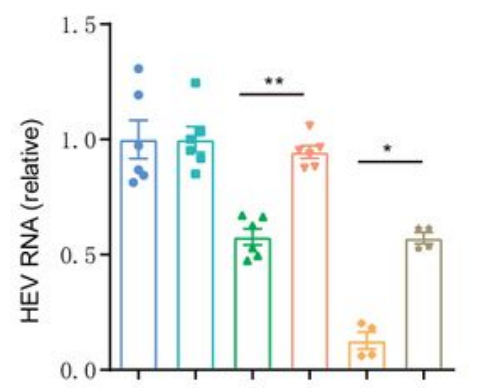

H

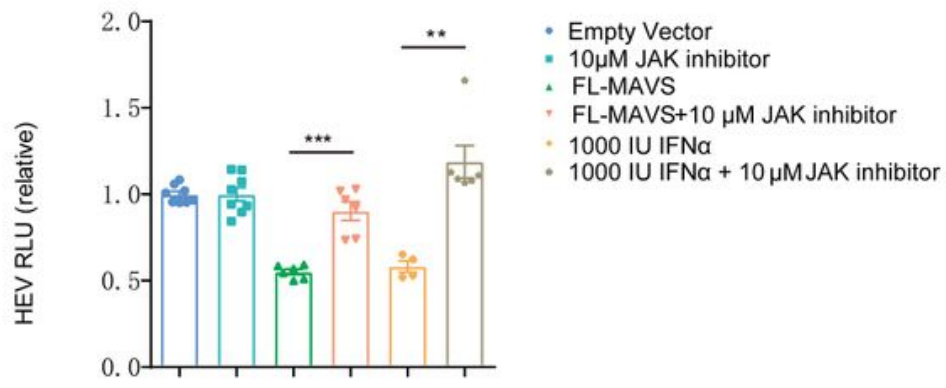

Figure 2

FL-MAVS restricts HEV via the JAK signaling Quantitative RT-PCR analysis of ISGs RNA in Huh7.5-p6 cell model transduced with FL-MAVS or empty vector $(n=2-3)$ for $48 \mathrm{~h}(\mathrm{~A})$. Quantitative RT-PCR analysis of IFNs RNA in Huh7.5-p6 cell model transduced with FL-MAVS or empty vector ( $\mathrm{n}=4-5)$ for $48 \mathrm{~h}$ (B). Huh7.5p6 cells were transduced with FL-MAVS or empty vector for $72 \mathrm{~h}$ and then the cells were washed 5 times and medium was refreshed. After another 72 h, supernatant was collected as conditioned medium. 
Analysis of firefly luciferase activity in Huh7-ISRE-Luc cells $(n=12)(C)$ or HCV replicon model $(n=12)(D)$ treated with Huh7.5-p6 derived conditioned medium for $72 \mathrm{~h}$. Huh7.5-p6 cell model transduced with FLMAVS or empty vector were treated with $1000 \mathrm{ng} / \mathrm{ml} 136 \mathrm{R}(\mathrm{n}=9-11)(\mathrm{E})$ or $1000 \mathrm{ng} / \mathrm{ml} \mathrm{B18R}(\mathrm{n}=13-14)(\mathrm{F})$ or $0.1 \%$ DMSO control for $48 \mathrm{~h}$. HEV RNA was measured by quantitative RT-PCR. Data in FL-MAVS group were presented relative to the empty vector group (set as 1). Data in the combination group of FL-MAVS with 136R or B18R were presented relative to 136R- or B18R-solely treated group (set as 1). Huh7.5-p6 model $(n=4-6)(G)$ and HEV replicon model $(n=4-8)(H)$ transduced with FL-MAVS or empty vector were treated with $10 \mu \mathrm{M}$ JAK inhibitor or 1000 IU IFNa or $0.1 \%$ DMSO vehicle control. Quantitative RT-PCR analysis of HEV RNA and luciferase activity were measured after $48 \mathrm{~h}$ treatment. Data in FL-MAVS group were presented relative to the empty vector group (set as 1). Data in the combination group of FL-MAVS with JAK inhibitor or IFNa were presented relative to JAK inhibitor or IFNa-solely treated group (set as 1). The above data are means \pm SEM $(* \mathrm{P}<0.05 ; * \star \mathrm{P}<0.01 ; * \star * \mathrm{P}<0.001)$.

\section{Supplementary Files}

This is a list of supplementary files associated with this preprint. Click to download.

- Supplementarymaterial.doc

- SupplementaryFigure1.tif 